\title{
Towards Sub-femtoscopic, Chromodynamic, Rutherford Scattering?
}

\author{
Francis Bench, John Dainton ${ }^{1}$ \\ Department of Physics \\ Oliver Lodge Laboratory \\ University of Liverpool, UK \\ E-mail: john.dainton@cockcroft.ac.uk
}

\begin{abstract}
Initial progress is reported of a phenomenological analysis in full view of chromodynamics of a plausible Bethe-Heitler-like approach to measurements of the virtual-photoproduction cross sections for vector-meson (VM) "quarkonia" (figure 1a) of different flavour, namely $\rho(760)$ $u \bar{u}-d \bar{d}, \phi(1019) s \bar{s}$, and $J / \psi(3096) c \bar{c}$. The cross section measurements by the H1 and Zeus experiments at the HERA electron-proton collider use exclusive electroproduction data $e p \rightarrow$ $e \mathrm{VM} p, N$ in which $N$ is an identifiable, colour-singlet, baryon system (resonant or non-resonant) with invariant mass $\lesssim 1.6 \mathrm{GeV}$. Their kinematic domain corresponds to the Regge asymptotic limit of the VM virtual-photoproduction mechanism. The Bethe-Heitler-like hypothesis includes the factorisable combination of virtual-photon structure with flavour-blind, elastic, quark-proton scattering - chromodynamic Rutherford scattering. The space-time "length" of elastic, quarkproton scattering is then determined by the space-like, "struck" quark of virtual-photon structure. Measurements by $\mathrm{H} 1$ of the proton structure function $F_{2}$ at low Bjørken- $x$ in the Regge asymptotic limit are used to calibrate the dependence of the forward elastic, quark-proton scattering amplitude on this "length". The measurements of the VM virtual-photoproduction cross sections are consistent with this Bethe-Heitler-like approach for plausible quark masses of flavour $u, d, s, c$, in which the elastic quark-proton scattering amplitude is attributable to a leading, dynamic, Regge pole, whose universality requires its trajectory to be dependent on both the space-time "length" and the 4-momentum transfer squared $(t)$ of the quark-proton interaction. As tradition demands, this Regge-pole may be taken as the pomeron, but now at work in a dimension below that of confinement and therefore below that of the interacting proton.
\end{abstract}

XXIV International Workshop on Deep-Inelastic Scattering and Related Subjects

11-15 April, 2016

DESY Hamburg, Germany

\footnotetext{
${ }^{1}$ Speaker

(c) Copyright owned by the author(s) under the terms of the Creative Commons

Attribution-NonCommercial-NoDerivatives 4.0 International License (CC BY-NC-ND 4.0).
} 


\section{Introduction}

First steps are here presented in a phenomenological analysis in full view of chromodynamics in terms of a plausible Bethe-Heitler-like [1] approach to measurements of the virtual-photoproduction cross sections for vector-meson (VM) "quarkonia" (figure 1a) of different flavour, namely $\rho(760) u \bar{u}-d \bar{d}$, $\phi(1019) s \bar{s}$, and $J / \psi(3096) c \bar{c}$, by the Zeus and H1 experiments at the HERA electron-proton collider.[2] The measurements are obtained from the observations of events which are kinematically consistent with the exclusive electroproduction of vector mesons

$$
e^{ \pm} p \rightarrow e^{ \pm} \mathrm{VMN}
$$

where

$$
\mathrm{VM}=\rho(770), \omega(782), \phi(1020), J / \psi(3096)
$$

is a vector meson of different "quarkonium" flavour $u, d, s, c$, and where $N$ is an identifiable, coloursinglet, baryon system (resonant or non-resonant) with invariant mass $\lesssim 1.6 \mathrm{GeV}$. No further distinction concerning the nature of $N$ is made in what follows. ${ }^{2}$

a)

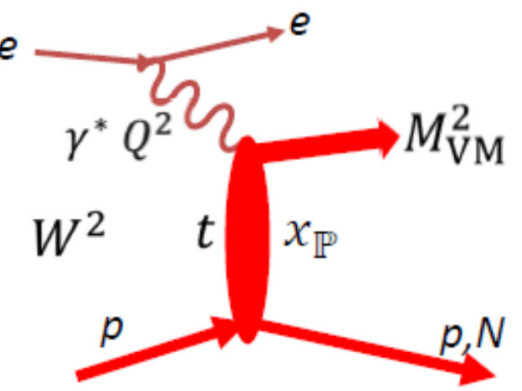

b) $e$

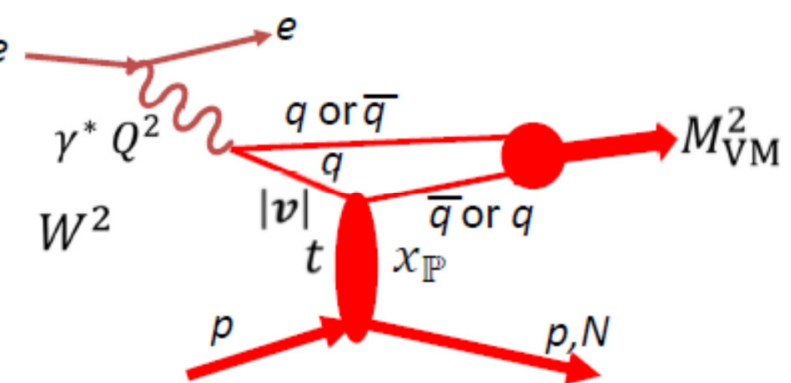

Figure 1: a) Schematic diagram illustrating the putative dynamics of the electroproduction in an interaction with 4momentum transfer squared $-Q^{2}$ of a vector meson VM "off" a proton $p$; the nature of the hadronic dynamics is setout here in terms of the invariant mass $W$, the invariant mass of the $\mathrm{VM} M_{\mathrm{VM}}$, the inelasticity $x_{\mathbb{P}}$ and the 4momentum transfer squared $t$ of the proton interaction; b) schematic diagram in terms of electron (positron), photon (virtual) and of the "pair-produced" QCD quanta quark $q$ and anti-quark $\bar{q}$ of a particular flavour; the $q$ or the $\bar{q}$ in the $q \bar{q}$ pair which is space-like with 4-momentum squared $v^{2}$ and therefore which exists within a space-time dimension of order $\frac{\hbar}{v}$, couples to the proton $p$ in an interaction with inelasticity $x_{\mathbb{P}}$ and 4-momentum transfer squared $t$ to produce the final state proton or proton excitation $N$ and a vector meson VM; the mechanism associated with b) defines a template for the kinematics which relate measured variables $Q^{2}, W^{2}, M_{\mathrm{VM}}^{2}$ and $t$ with sub-confinement variables virtuality $v^{2}$ of $q$ or $\bar{q}$ and the quark mass $M_{q}$, and with which measurements of the virtual photoproduction $\gamma^{*} p \rightarrow V M p, N$ of $q \bar{q}$ "onium" VM with different quark flavour can be compared.

The aim is to develop a phenomenological approach to the analysis of such measurements which exposes the chromodynamics of elastic $(q p \rightarrow q p)$ and "elastic dissociative" $(q p \rightarrow q N)$ quark-proton (and anti-quark-proton) scattering, that is, which exposes the simplest two-to-two body (Rutherford) scattering in sub-femtoscopic (sub-confined) QCD with no transfer of colour between interacting quark and proton - in other words chromodynamic Rutherford scattering. Just as Bethe-Heitler $e^{+} e^{-}$pair production is readily interpreted in terms of the embedding of elastic virtual electron-proton $\left(e^{*} p \rightarrow e p\right)$ scattering with electrodynamic photon splitting $\left(\gamma \rightarrow e^{ \pm} e^{*}\right)$, an interpretation of VM virtualphotoproduction is sought in terms of the embedding of elastic quark-proton $\left(q^{*} p \rightarrow q p\right)$ scattering with leading order, flavour-sensitive, photon splitting $\gamma^{*} \rightarrow q^{ \pm} q^{* 3}$ (figure 1b) in which also the final $q \bar{q}$ mass is constrained to have the mass (spectrum) of a vector meson.

\footnotetext{
${ }^{2}$ Thus, the proton $p$ can interact elastically or inelastically leading to an isopin- $\frac{1}{2}$ baryon excitation of mass $<1.6$ $\mathrm{GeV}$ which is consistent with vacuum dominance of the $t$-channel in the virtual-photon $N$ interaction; resonant candidates include $N(938) \frac{1}{2}^{+}$(proton $\left.p\right), N(1440) \frac{1}{2}^{+}$.

${ }^{3}$ The superscript $*$ is here used universally to specify a virtual (off-shell) quantum, either space-like or time-like, either boson or fermion.
} 
The methodology here is experimental. It follows a prescription in which the measurement of a cross section for exclusive process such as (1) can be investigated further in terms of the variables quark mass and quark virtuality which are important for a chromodynamic understanding.

\section{Measurements}

The available measurements lie in the kinematic domain $10 \lesssim W \lesssim 300 \mathrm{GeV}, 1 \lesssim Q^{2} \lesssim 40 \mathrm{GeV}^{2}$, $|t| \ll 1 \mathrm{GeV}^{2}$, corresponding well to the Regge-asymptotic limit [3]

$$
\frac{W^{2}+Q^{2}-m_{p}^{2}}{M_{\mathrm{VM}}^{2}+Q^{2}-t} \rightarrow \infty
$$

that is to $W^{2} \gg Q^{2} \gtrsim m_{p}^{2}>|t| \sim 0$. At fixed $Q^{2}$ the $\mathrm{VM}$ virtual-photoproduction cross sections $\sigma_{\gamma^{*} p \rightarrow \mathrm{VM} p, N}$ for the production of the vector mesons (VM) $\rho(760), \phi(1019)$ and $J / \psi(3096)$ are observed to increase modestly with increasing centre-of-momentum energy $W$. These modest increases depend both on $Q^{2}$ and on "quarkonium" flavour $u / d, s, c$ and are well quantified by $\delta\left(Q^{2}\right)$ in a dependence $\propto W^{\delta\left(Q^{2}\right)}$. The measurements of $\delta\left(Q^{2}\right)$ which are used in what follows are in Table 1 .

\section{Bethe-Heitler-like Hypothesis}

The cross sections thus exhibit the characteristics associated with those of elastic and dissociative hadronic interactions in which, as well as a modest increase with increasing $W$, no hypercharge is possible in the $t$-channel and the target proton interacts peripherally so that $t \sim 0$. The $W^{2}$-dependences and their dependences on $Q^{2}$, which are encapsulated in $\delta\left(Q^{2}\right)$, suggest that the hypothesis of a single leading Regge-pole with a single trajectory $\alpha_{\mathbb{P}}(t)$, traditionally taken to be that of the pomeron $\mathbb{P}$ as first proposed in elastic and dissociative diffractive hadronic interactions, is not tenable. Instead, $\alpha_{\mathbb{P}}\left(Q^{2}, t\right)$ must have a dependence on $Q^{2}$ as well as on $t$, something which can still be acceptable within the framework of Regge theory if the necessary analyticity is now to be found seperately in each of the (infinite) set of production scattering amplitudes, one for each $Q^{2}$.

The dependence on quark flavour (hypercharge) of any electromagnetic process such as VM electroproduction is of course to be expected and is self-evident. The flavourblindness of chromodynamics is a matter hitherto of overwhelming experimental verification. It is therefore appropriate to proceed to an understanding of VM electroproduction with the assumption of a Bethe-Heitler-like mechanism in which, in the Regge-asymptotic limit, an elastic or dissociative hadronic interaction (here any one of $\left.q^{*} p \rightarrow q p, q=u, d, s, c\right)$ is described by the leading, flavour-blind, Regge pole, the pomeron $\mathbb{P}$. This elastic scattering is embedded in a factorisable manner together with standard, hypercharge-specified, quark coupling to the virtual photon $\gamma^{*}$. Such a mechanism corresponds to a chromodynamic version of the Bethe-Heitler process in electrodynamics (figure 1b). The quark coupling to the virtual photon $\gamma^{*}$ is taken to be that corresponding to measurements of the virtual-photon structure function $F_{2}^{\gamma^{*}}\left(x, Q^{2}\right)$.

Experiment has demonstrated that $F_{2}^{\gamma^{*}}$ is

\begin{tabular}{|c|c|c|}
\hline Interaction & $\begin{array}{c}Q^{2} \\
\left(\mathrm{GeV}^{2}\right)\end{array}$ & $\begin{array}{c}\delta \text { (士stat. } \pm \text { syst.) } \\
\sigma_{\gamma^{*} p \rightarrow \mathrm{VM}, N} \propto W^{\delta}\end{array}$ \\
\hline & 3.3 & $0.4 \pm 0.08 \pm 0.06$ \\
$\gamma^{*} p \rightarrow \rho(0.76) p$ & 6.6 & $0.57 \pm 0.11 \pm 0.06$ \\
& 11.9 & $0.28 \pm 0.15 \pm 0.05$ \\
& 19.5 & $0.77 \pm 0.15 \pm 0.05$ \\
& 35.6 & $1.17 \pm 0.26 \pm 0.04$ \\
\hline & 3.3 & $0.32 \pm 0.17_{-0.09}^{+0.08}$ \\
$\gamma^{*} p \rightarrow \rho(0.76) N$ & 7.5 & $0.17 \pm 0.14_{-0.09}^{+0.07}$ \\
& 22.5 & $0.58 \pm 0.29_{-0.13}^{+0.10}$ \\
\hline & 3.3 & $0.53 \pm 0.04 \pm 0.02$ \\
$\gamma^{*} p \rightarrow \phi(1.019) p$ & 6.6 & $0.52 \pm 0.05 \pm 0.02$ \\
& 15.8 & $1.09 \pm 0.08 \pm 0.02$ \\
\hline$\gamma^{*} p \rightarrow \phi(1.019) N$ & 5 & $0.50 \pm 0.24_{-0.20}^{+0.16}$ \\
\hline & 0.1 & $0.67 \pm 0.03$ \\
& 0.05 & $0.75 \pm 0.03 \pm 0.03$ \\
$\gamma^{*} p \rightarrow \psi(3.1) p$ & 3.2 & $0.67 \pm 0.02 \pm 0.14$ \\
& 7.0 & $0.83 \pm 0.31 \pm 0.15$ \\
& 22.4 & $0.69 \pm 0.32 \pm 0.14$ \\
\hline
\end{tabular}

Table 1: The set of measurements at HERA of cross sections $\sigma_{\gamma^{*} p \rightarrow \mathrm{VM} p, N}$ for the virtual-photoproduction of vector mesons of different "quarkonium" flavour $u, d, s$, $c$; here $p$ is a proton and $N$ is an identifiable, coloursinglet, baryon system (resonant or non-resonant) with invariant mass $\lesssim 1.6 \mathrm{GeV}$; measurements of the $W$-dependences of the total cross-sections at each $Q^{2}$ are fitted to a form $W^{\delta}$ with the values of $\delta$ tabulated. 
well-described in QCD to at least NLO as long as the space-like $\gamma^{*}$ has invariant mass squared $-P^{2} \lesssim$ $-0.25 \mathrm{GeV}^{2}$.[4] It has also demonstrated that a phenomenlogical description is possible by taking leading order flavour-dynamic splitting $\gamma^{*} \rightarrow q^{*} \bar{q}$ or $\gamma^{*} \rightarrow \bar{q}^{*} q(q=u, d, s, c)$ in which each flavour of quark has a "constituent mass", that is, each quark has a mass consistent with the requirement that the valence quarks alone can account for its hadronic spectroscopy.

The $\mathbb{P}$ trajectory $\alpha_{\mathbb{P}}^{q p}\left(v^{2}, t\right)$ of the embedded $q^{*} p \rightarrow q p$ interaction is then a function, as well as of $t$, of the virtuality $v^{2}$ of the initial, space-like, virtual quark $q^{*}$ (or anti-quark $\bar{q}^{*}$ ), that is, $\alpha_{\mathbb{P}}^{q p}\left(v^{2}, t\right)$ is then a function of the space-time "length" $\Delta=\frac{\hbar}{|v|}$ of the $q^{*} p \rightarrow q p$ (or $\bar{q}^{*} p \rightarrow \bar{q} p$ ) interaction when observed from a frame in which $q^{*}$ and proton $p$ appear to be collinear. ${ }^{4}$ The observed $Q^{2}$-dependence of the rising $W$-dependences $\propto W^{\delta\left(Q^{2}\right)}$ of the cross sections $\sigma_{\gamma^{*} p \rightarrow \mathrm{VM} p, N}$, that is of $\delta\left(Q^{2}\right)$, for each flavour of "quarkonia" is then considered to be a consequence of the dependences

$$
\frac{\mathrm{d} \sigma_{q^{*} p \rightarrow q p}}{\mathrm{~d} t} \propto s_{q p}^{2 \alpha_{\mathbb{P}}^{q p}\left(v^{2}, t\right)-2}
$$

on centre-of-momentum energy squared $s_{q p}$ of the differential cross sections for elastic $q^{*} p \rightarrow q p$ or $\bar{q}^{*} p \rightarrow \bar{q} p$ scattering of given initial virtuality $v^{2}$. The flavour-sensitivity of $\sigma_{\gamma^{*} p \rightarrow V M p, N}$ then arises only through the flavour-sensitivity of the virtual-photon splitting.

Without then having to make any further assumptions which would be the case if the Bethe-Heitlerlike production mechanism for the cross section is modelled, it is possible to test such a proposal by recognising that the virtuality $v^{2}$ of the initial space-like, virtual quark $q^{*}$ (or anti-quark $\bar{q}^{*}$ ) must lie within limits

$$
0 \geq v_{\min }^{2} \geq v^{2} \geq v_{\max }^{2}
$$

which, it can easily be seen, are determined by $Q^{2}, t \sim 0$, constituent quark mass $M_{q}$ and vector-meson "quarkonium" mass $M_{\mathrm{VM}}$ as

$$
M_{q}^{2}-v_{\max }^{2}=\frac{M_{\mathrm{VM}}^{2}+Q^{2}-t}{2}\left(1 \mp \sqrt{1+4 \frac{Q^{2} t}{\left(M_{\mathrm{VM}}^{2}+Q^{2}-t\right)^{2}}} \cdot \sqrt{1-4 \frac{M_{q}^{2}}{M_{\mathrm{VM}}^{2}}}\right) .
$$

Then also is the trajectory $\alpha_{\mathbb{P}}^{q p}\left(v^{2}, t \sim 0\right)$ of the leading Regge pole $\mathbb{P}$ in the $q^{*} p \rightarrow q p$ elastic scattering confined to be within the corresponding limits

$$
\alpha_{\mathbb{P}}^{q p}\left(v_{\text {min }}^{2}, t \sim 0\right) \leq \alpha_{\mathbb{P}}^{q p}\left(v^{2}, t \sim 0\right) \leq \alpha_{\mathbb{P}}^{q p}\left(v_{\text {max }}^{2}, t \sim 0\right)
$$

which are also determined by $Q^{2}, t \sim 0, M_{q}$ and $M_{\mathrm{VM}}$. There follows then a prediction of this BetheHeitler-like approach for the measurements of the $W$-dependences of the cross sections $\sigma_{\gamma^{*} p \rightarrow \mathrm{VM} p, N}$, namely that $\delta\left(Q^{2}\right)$ in the fitted $W^{\delta\left(Q^{2}\right)}$-dependences must lie between the limits

$$
4 \alpha_{\mathbb{P}}^{q p}\left(v_{\text {min }}^{2}, t \sim 0\right)-4 \lesssim \delta\left(Q^{2}\right) \lesssim 4 \alpha_{\mathbb{P}}^{q p}\left(v_{\text {max }}^{2}, t \sim 0\right)-4 .
$$

Here the approximate nature of this restriction arises because of the fact that the effect of any dependence of the trajectory $\alpha_{\mathbb{P}}^{q p}\left(v^{2}, t\right)$ on $t$ is taken to be small, which is the case because the proton interacts peripherally.

For these limits, the trajectory $\alpha_{\mathbb{P}}^{q p}\left(v^{2}, t\right)$ is defined (precisely at $t=0$ ) by means of the dependence at fixed $Q^{2}$ of measurements of the proton structure function $F_{2}\left(x_{\mathrm{Bj}}, Q^{2}\right)$ on Bjørken- $x$

$$
x_{\mathrm{Bj}}=\frac{Q^{2}}{W^{2}+Q^{2}} \text {. }
$$

\footnotetext{
${ }^{4}$ That the Regge asymptotic limit $x_{\mathbb{P}} \rightarrow 0$, where $x_{\mathbb{P}}$ is the inelasticity at the incident proton of the observed virtualphotoproduction cross section $\sigma_{\gamma^{*} p \rightarrow \mathrm{VM} p, N}$, also corresponds to the same limit of the embedded elastic quark-proton scattering cross section $\sigma_{q^{*} p \rightarrow q p, N}$ follows from the duality of definitions of $x_{\mathbb{P}}$ at the incident proton. In an obvious 4vector notation, for respectively $\gamma^{*} p \rightarrow \operatorname{VM} p, N$ and $q^{*} p \rightarrow \mathrm{q} p, N$ we have

$$
x_{\mathbb{P}}=\frac{\mathbb{P} \cdot \gamma^{*}}{p \cdot \gamma^{*}}=\frac{M_{\mathrm{VM}}^{2}+Q^{2}-t}{W^{2}+Q^{2}-t} \quad x_{\mathbb{P}}=\frac{\mathbb{P} \cdot q^{*}}{p \cdot q^{*}}=\frac{M_{q}^{2}-v^{2}-t}{s_{q p}-v^{2}-t} .
$$

In the Regge limit of $\gamma^{*} p \rightarrow \operatorname{VM} p, N$, this duality of expressions then requires

$$
\frac{M_{\mathrm{VM}}^{2}+Q^{2}-t}{W^{2}+Q^{2}-t}=\frac{M_{q}^{2}-v^{2}-t}{s_{q p}-v^{2}-t} \underset{t \rightarrow 0}{\longrightarrow} \frac{M_{\mathrm{VM}}^{2}+Q^{2}}{W^{2}+Q^{2}}=\frac{M_{q}^{2}-v^{2}}{s_{q p}-v^{2}} \underset{t \rightarrow 0, W^{2} \gg Q^{2}, M_{\mathrm{VM}}^{2}}{\longrightarrow} 0
$$

demonstrating also therefore that this limit also includes the Regge limit of the quark elastic scattering $q^{*} p \rightarrow q p, N$.
} 
Because the cross section

$$
\sigma_{\gamma^{*} p}\left(W^{2}, Q^{2}\right)=\frac{4 \pi^{2} \alpha}{Q^{2}} F_{2}\left(x_{\mathrm{Bj}}, Q^{2}\right)
$$

describes totally inclusive inelastic $e p \rightarrow e X$ interactions, the optical theorem can be applied to relate either $\sigma_{\gamma^{*}}$ or $F_{2}$ to the forward $(t=0)$, elastic, $\gamma^{*} p \rightarrow \gamma^{*} p$ scattering amplitude (figure 2). Further, because the QFD interactions $e u^{*} \rightarrow e u$ and $e d^{*} \rightarrow e d$ between lepton probe and $u$ and $d$-quarks in proton structure predominate in inclusive inelastic $e p \rightarrow e X$ interactions, this $\gamma^{*} p \rightarrow \gamma^{*} p$ scattering amplitude can be written as the combination of the chromodynamic splitting to, or if preferred the chromodynamic fusion of, quarks $\gamma^{*} \leftrightarrow u^{*} \bar{u}, \bar{u}^{*} u$ and $\gamma^{*} \leftrightarrow d^{*} \bar{d}, \bar{d}^{*} d$ and the corresponding forward elastic scattering $q^{*} p \rightarrow q^{*} p$ (figure 3). Further, to the extent that the quark mass squared (predominantly $u$ and $d$ in a proton) is negligible when compared with $Q^{2}$, the virtuality $v^{2}$ of the quark $q^{*}$ or anti-quark $\bar{q}^{*}$ is then just $Q^{2}{ }^{5}$ If the $q^{*} p \rightarrow q^{*} p$ elastic scattering is in the Regge-asymptotic domain, that is, the kinematic domain of the measurements of the total $\gamma^{*} p$ cross section $\sigma_{\gamma^{*} p}\left(W^{2}, Q^{2}\right)$ is consistent with

$$
\frac{1}{x_{\mathrm{Bj}}}=\frac{W^{2}+Q^{2}}{Q^{2}} \rightarrow \frac{W^{2}}{Q^{2}} \rightarrow \infty,
$$

then the structure function at fixed $Q^{2}$ is approximated by the dependence

$$
F_{2}\left(x_{\mathrm{Bj}}, Q^{2}\right) \propto x_{\mathrm{Bj}}^{-\lambda\left(Q^{2}, t=0\right)} \underset{W^{2} \gg Q^{2}, m_{p}^{2}}{\longrightarrow} W^{2 \lambda\left(Q^{2}, t=0\right)}
$$

where

$$
\lambda\left(Q^{2}, t=0\right)=\alpha_{\mathbb{P}}^{q p}\left(Q^{2}, t=0\right)-1 .
$$

The fits by the $\mathrm{H} 1$ experiment [5] of this form to measurements of $F_{2}\left(x_{\mathrm{Bj}}, Q^{2}\right)$ give the logarithmic form

with

$$
\lambda\left(Q^{2}, t=0\right)=a \ln \frac{Q^{2}}{\Lambda^{2}}
$$

and

$$
a=0.0481 \pm 0.0013 \text { (stat) } \pm 0.0037 \text { (syst) }
$$

$\Lambda=0.292 \pm 0.020$ (stat) \pm 0.0051 (syst) GeV,

which can then be taken to be a determination of the trajectory intercept

$$
\lambda\left(Q^{2}, t=0\right)=\alpha_{\mathbb{P}}^{\gamma^{*} p \rightarrow \gamma^{*} p}\left(Q^{2}, t=0\right)-1 \rightarrow \lambda\left(v^{2}, t=0\right)=\alpha_{\mathbb{P}}^{q^{*} p \rightarrow q p}\left(v^{2}, t=0\right)-1
$$

as a function of $Q^{2}$, and therefore of $v^{2}$. This specification of $\lambda\left(v^{2}, t=0\right)$ then amounts to a precise determination of the elastic $\gamma^{*} p \rightarrow \gamma^{*} p$ scattering amplitude in its Regge-asymptotic domain as a function of the length $\Delta=\frac{\hbar}{|v|}$ of the embedded $q^{*} p \rightarrow q^{*} p$ (or $\bar{q}^{*} p \rightarrow \bar{q}^{*} p$ ) interaction when observed from a frame in which $q^{*}$ (or $\bar{q}^{*}$ ) and $p$ appear collinear.

\section{Results}

This elastic $\gamma^{*} p \rightarrow \gamma^{*} p$ scattering amplitude in its Regge-asymptotic domain, and specifically the discrepancy $\lambda\left(Q^{2}, t=0\right)$ of the trajectory which emerges from the analysis of the proton structure function $F_{2}$, can then be used to determine the limits (in the form of limits on $\delta\left(Q^{2}\right)$ discussed above and given by $4 \lambda\left(Q^{2}, t=0\right)$ ). The $W$-dependences as a function of $Q^{2}$ for each flavour of the virtual photoproduction cross sections for VM "quarkonia" must lie between these limits if there is to be consistency with the Bethe-Heitler approach.

\footnotetext{
${ }^{5}$ This virtuality is calculated by considering the rest-frame of the invariant mass squared $M_{q}^{2}$ of the time-like, quark $q$ in figure $3 \mathrm{~b}$ ). In this frame the decay products must themselves have equal magnitude of momentum $p^{*}$ and thus each must have the individual positive energies

$$
E_{\gamma}^{*}=\frac{M_{q}^{2}-Q^{2}-v^{2}}{2 M_{q}}>0 \quad E_{v}^{*}=\frac{M_{q}^{2}+v^{2}+Q^{2}}{2 M_{q}}>0 .
$$

It immediately follows that the virtuality $v^{2}$ of the space-like quark must lie in a range defined by $M_{q}^{2}>v^{2}+Q^{2}>-M_{q}^{2}$,

so that, when the constituent mass is small, which is the case in deep-inelastic scattering $e p \rightarrow e X$ where current $u$ and $d$ quarks predominate, the space-like virtuality $v^{2}$ is $-Q^{2}$ (as is of course to be expected).
} 


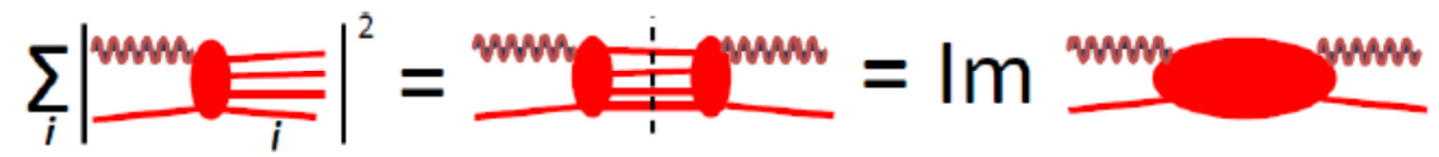

Figure 2: The optical theorem "in a picture" for deep-inelastic lepton-proton scattering interpreted in terms of inelastic virtual photon-proton $\left(\gamma^{*} p\right)$ interactions: the total $\gamma^{*} p$ (hadronic virtual- photoproduction) cross-section equals the imaginary part (thus the vertical dotted line to indicate that the sum over final states corresponds to onshell observable states) of the forward (zero scattering angle, 4-momentum transfer squared $t=0$ ) elastic (and therefore diffractive) $\gamma^{*} p$ scattering amplitude, that is the forward proton Compton scattering amplitude.

Figure 4 summarises the results in the form of "wedge-shaped" surfaces corresponding to the limits on $\delta \equiv 4 \lambda\left(v^{2}, t=0\right)$, or equivalently on the length $\Delta=\frac{\hbar}{|v|}$ of the embedded $q^{*} p \rightarrow q p$ (or $\bar{q}^{*} p \rightarrow \bar{q} p$ ) interaction when observed from a frame in which $q^{*}$ or $\bar{q}^{*}$ and $p$ appear collinear, for varying $Q^{2}$ and quark mass $M_{q}$. For consistency with the Bethe-Heitler approach, the "wedge" must sandwich measurements of $\delta\left(Q^{2}\right)$ for VM virtual photoproduction of each flavour of "quarkonium", and if it does, then this consistency may also constrain the quark mass $M_{a}$ with this flavour.

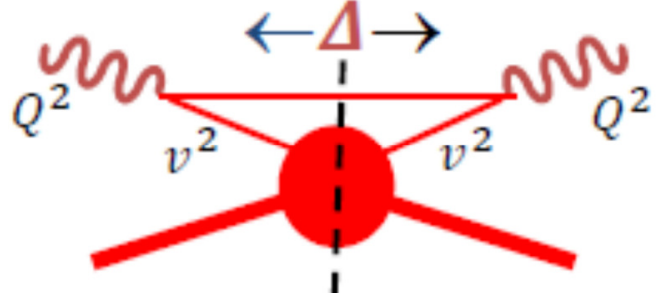

Figure 3: Forward virtual-photon Compton scattering in which the (well known) coupling of the space-like photon of invariant mass squared $-Q^{2}$ is to a time-like, "on-shell" (anti-)quark and a space-like (quark)anti -quark; the internal summation of the optical theorem is now therefore over all possible configurations of this time-like (anti)quark and incident and emerging time-like proton within external, space-like propagating photons of invariant mass squared $-Q^{2}$.

The lack of evidence for any $Q^{2}$-dependence of $\delta$ for $J / \psi(3096)$ virtual-photoproduction is seen in figure 4a) to be such that the measurements lie within the limits for a range of charm quark mass $M_{q=c}$ up to about $1.1 \mathrm{GeV} / c^{2}$, which is consistent with the accepted value of the quantum flavour-dynamic (QFD) current mass of the charm-quark. Given the mass of the $J / \psi(3096)$ for this upper limit on $M_{q=c}$, there is relatively little variation of quark virtuality $v^{2}$ possible so that the length $\Delta$ of the embedded $c^{*} p \rightarrow c p$ elastic scattering is always $\sim 0.08 \mathrm{fm}$. This makes good physics common sense!

In contrast in figure $4 \mathrm{~b}$ ) are shown the $Q^{2}$-dependent measurements of $\delta$ for $\rho(760)$ virtualphotoproduction. Now the contour ("wedge") which is defined by the limits admits values of constituent quark ( $u$ and $d$ ) mass $M_{q=u / d}$ up to about $0.2 \mathrm{GeV} / c^{2}$ at which value they are drawn in figure $5 \mathrm{~b}$ ) and above which the measurements at lower $Q^{2}$ begin to fall outside the surface for the lower limit. For this consistency up to the limit on $M_{q=u / d} \sim 0.2 \mathrm{GeV} / c^{2}$ the observed $Q^{2}$-dependence of $\delta$ is accounted for by more than an order of magnitude range of lengths $\Delta$ of the $u^{*} / d^{*} p \rightarrow u / d p$ elastic scattering between $\sim 0.7 \mathrm{fm}$ and $\sim 0.09 \mathrm{fm}$.

In figure 4c) are shown the measurements of $\delta$ for $\phi(1019)$ electroproduction. Two perspectives of the dependences on $Q^{2}$ and $M_{q=s}$ of $\delta$ are displayed; the measurements are shown first in the plane corresponding to low $M_{q=s}=0.1 \mathrm{GeV}$ and second in the plane corresponding to high $M_{q=s}=0.5 \mathrm{GeV}$. The "sandwich" of limits is everywhere seen to include the measurements of $\delta\left(Q^{2}\right)$, albeit within substantial error. The accuracy is insufficient to make any statement concerning presence or absence of any $Q^{2}$-dependence. The precision with which there is consistency therefore amounts to contributions of length $\Delta$ of $s^{*} p \rightarrow s p$ elastic scattering which again can vary by more than an order of magnitude range between $\sim 0.7 \mathrm{fm}$ and $\sim 0.09 \mathrm{fm}$.

Both of the upper limits on quark masses, $M_{u, d} \lesssim 0.2 \mathrm{GeV}$ and $M_{c} \lesssim 1.2 \mathrm{GeV}$, coincide roughly with expectation, for the light $u / d$-quarks with determinations in for example photon structure and hadron spectroscopy of "constituent mass", and for the $c$-quark with flavour-dynamic "current" mass. For $\phi$ (1019) $s \bar{s}$ there is no evidence for any inconsistency for any allowed choice of mass $0 \leq M_{s} \leq$ 
$0.5 \mathrm{GeV} \sim \frac{M_{\phi}}{2}$, though it is interesting to note that there is a marginally visible, but not statistically significant, preference for $M_{s} \sim 0.4 \mathrm{GeV}$, that is for a mass which is larger than the upper limit on $u / d$ quarks.
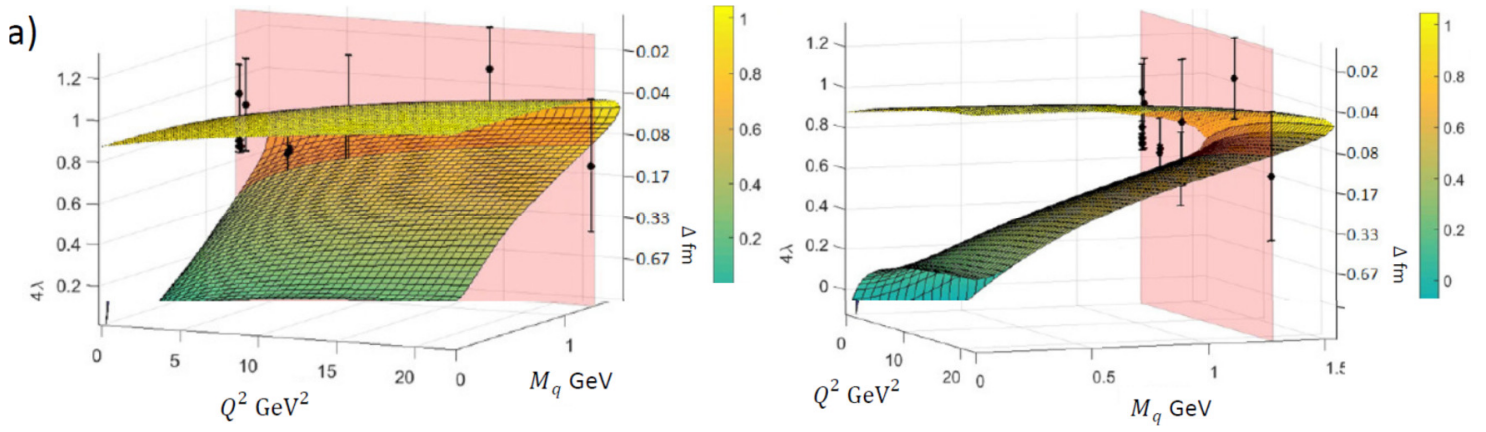

b)
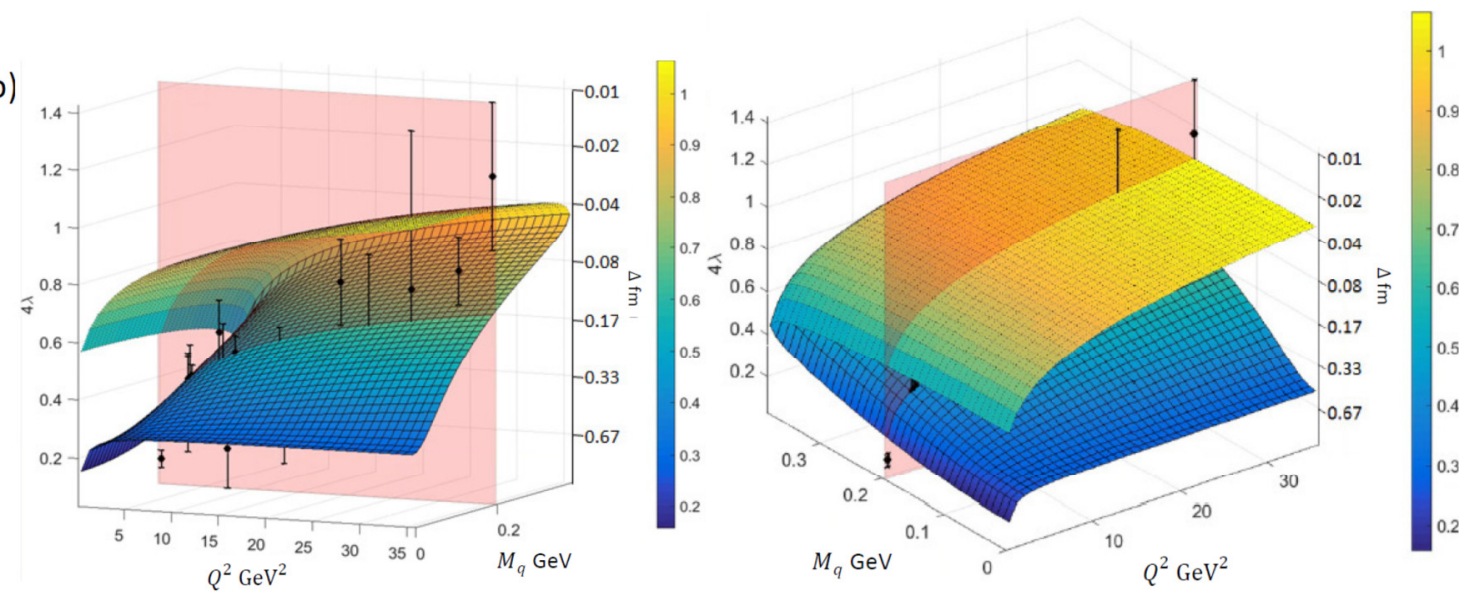

c)
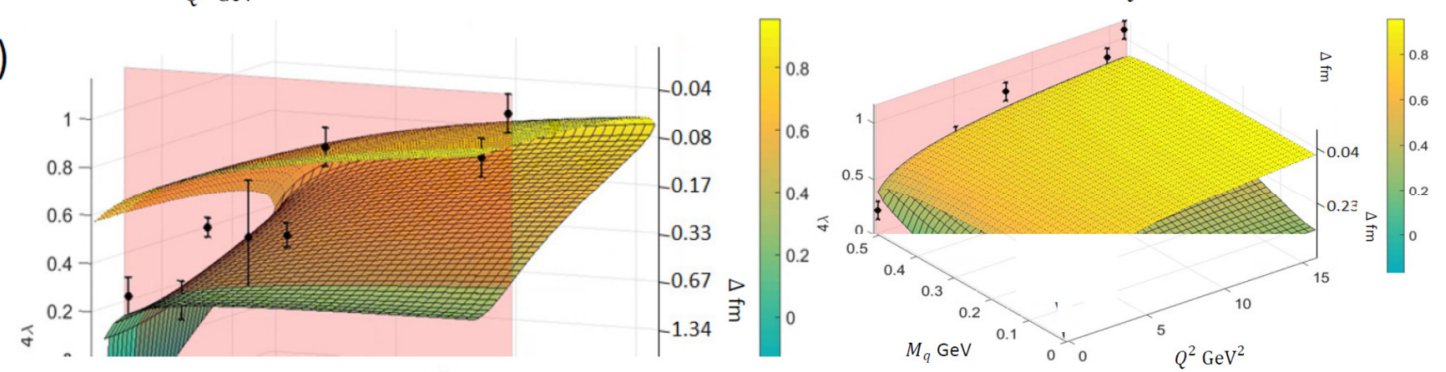

Figure 4: In the three-dimensional domain of the variables 4-momentum transfer squared $Q^{2}$, quark mass $M_{q}$, and the parameter $\delta\left(Q^{2}\right)$ where the latter is obtained from fits of the form $\sigma_{\gamma^{*} p \rightarrow \mathrm{VM} p, N}\left(W^{2}, Q^{2}\right) \propto W^{\delta}$ to the $W$-dependence at fixed $Q^{2}$ is shown for each of three flavours of VM "quarkonium"; the "wedge", inside of which is the region of allowed values of $\delta$, folds at the kinematic limit $M_{q}=\frac{M_{\mathrm{VM}}}{2}$; the wedge surface is determined assuming upper and lower limits on $\delta\left(Q^{2}\right)$ which correspond to the range of values of the virtuality $\left(v^{2}\right.$ and equation (2) in the text) of the space-like quark $q^{*}$ (or $\bar{q}^{*}$ ) in the initial virtual photon splitting $\gamma^{*} \rightarrow q^{*} \bar{q}$ (or $\bar{q}^{*} q$ ) which is embedded in the chromodynamic Bethe-Heitler-like diagram figure 1b); the vertical axis $\delta\left(Q^{2}\right)$ is also labelled as $4 \lambda=4 \alpha_{\mathbb{P}}^{q p}-4$ where $\alpha_{\mathbb{P}}^{q p}\left(v^{2}, t=0\right)$ is the intercept of the trajectory of the leading Regge pole in the $q p$ elastic scattering which is also embedded in figure $1 \mathrm{~b}$ ), and also as $\Delta(\mathrm{fm})$ which is the space-time length of the $q p$ elastic scattering when viewed from a frame in which initial $q^{*}$ and $p$ are collinear; the measurements of $\delta\left(Q^{2}\right)$ for each of the quarkonia flavours $u / d, s, c$ which are displayed are from Table 1); a) for $\sigma_{\gamma^{*} p \rightarrow J / \psi p, N}\left(W^{2}, Q^{2}\right), \delta\left(Q^{2}\right)$ for $J / \psi(3096) c \bar{c}$ virtual-photoproduction; the measurements are shown in the plane of constant $M_{q}$ at the highest value $\left(M_{q} \sim 1.1 \mathrm{GeV}\right)$ which is consistent with being within the wedge of limits; b) as a) for $\sigma_{\gamma^{*} p \rightarrow \rho p, N}\left(W^{2}, Q^{2}\right), \delta\left(Q^{2}\right)$ for $\rho(760)$ $u \bar{u}-d \bar{d}$ virtual-photoproduction; the measurements are again shown for the highest value $\left(M_{q} \sim 0.2 \mathrm{GeV}\right)$ which is consistent with being within the wedge of limits; c) as a) for $\sigma_{\gamma^{*} p \rightarrow \phi p, N}\left(W^{2}, Q^{2}\right), \delta\left(Q^{2}\right)$ for $\phi(1019) s \bar{s}$ virtualphotoproduction; the measurements are everywhere within error within the within the wedge of limits for all $M_{q}$ and are therefore shown for low $M_{q}=0.1 \mathrm{GeV}\left(M_{q}\right.$ axis not visible) and high $M_{q}=0.5 \mathrm{GeV}$.

There is a further corollary to these conclusions on quark mass for each quark flavour when it is appreciated that, when the mass of the quark-antiquark pair is not constrained to be that of a meson or 
meson resonance, inclusive chromodynamic Bethe-Heitler-like production amounts to deeply inelastic diffraction $e p \rightarrow e X p, N$. Then the limits for significant $Q^{2}$ in equation (2) can admit a wide range $\sim 0 \geq v^{2} \geq v_{\max }^{2}$ of quark virtuality, therefore a wide range of quark-proton interaction "length" $\Delta$, and therefore a wide range of intercept of dynamic pomeron trajectory $\alpha_{\mathbb{P}}^{q p}\left(v^{2}, t=0\right)$. Because of this, the controversy in the 1990s concerning the observation or otherwise of a "hard pomeron" in deeply inelastic diffractive electron scattering can now be seen to have been entirely futile. It is the "decay $q$-value" of the constituent quark-antiquark pair in its "quarkonium" which determines the contributing range of quark virtuality $v^{2}$ in the embedded quark-proton elastic scattering and which may, if this $q$-value is small, restrict $|v|$ to larger values. For then the quark-proton scattering is "shorter" so that the $W$-dependence of $\sigma_{\gamma^{*} p \rightarrow \mathrm{VM} p, N} \propto W^{\delta}$ is steeper, that is, $\delta$ is larger.

\section{Conclusions}

Initial preliminary evidence has been presented from measurements of the exclusive virtual photoproduction of vector mesons at high energy that the production mechanism follows the simplest expectation of a chromodynamic Bethe-Heitler-like process in which the quark and anti-quark are confined in the "quarkonium" of appropriate flavour, $\rho(760) u \bar{u}-d \bar{d}, \phi(1019) s \bar{s}$ and $J / \psi(3096) c \bar{c}$.

The masses of quarks $u / d, s, c$ are found to be consistent with being between presently accepted "current" and "constituent" values.

The embedded quark-proton (or anti-quark proton) elastic scattering cross section is observed to follow the expectation which is derived from the interpretation of deeply-inelastic, inclusive, electronproton scattering, in particular the application of the optical theorem to the proton structure function $F_{2}$. In the Regge-asymptotic limit at low Bjørken- $x, F_{2}$ is well known to be described in terms of forward elastic virtual-photon Compton scattering by the dominance of a single, leading, universal, Regge pole. This pole is consistent with a modestly increasing dependence on CM energy squared, it requires a trajectory which is dependent on $Q^{2}$, and, following the coupling with a $u$ or $d$-quark of the deeply-inelastic, flavourdynamic, probe, it specifies the forward, elastic. $u$ or $d$-quark proton scattering amplitude. If then tradition is followed and this Regge pole is taken to be the pomeron $\mathbb{P}$, its universality, and therefore its utility as demonstrated here in its application to vector meson virtual-photoproduction, require that it be dynamic with a trajectory which depends on the space-like virtuality of the elastically scattering $u$ or $d$ quark as well as on the 4-momentum transfer squared $t$ of the elastic $u$ or $d$-quark-proton interaction. When observed from a frame in which the interaction is collinear, this dependence on virtuality of the trajectory can be interpreted as a dependence of the scattering amplitude for chromodynamic Rutherford scattering on the length $\Delta$ of the elastic, quark-proton interaction.

Theoretically, such an outcome in terms of a universal dynamic pomeron can be anticipated in chromodynamics if the pioneering calculations by Lipatov and collaborators [6] of quark-quark elastic scattering are also applicable in quark-proton elastic scattering. This is likely to be the case because the space-time "length" of this elastic scattering must be, and here is always found to be, below the confinement dimension when taken to be that of the size of the proton.

Experimentally, the supposition of a single, universal, dynamic pomeron, the role of quark virtuality in its trajectory, and the interpretation of this dependence on virtuality when observed in a collinear frame perhaps merit the replacement of the terminology of "harder" and "softer" diffraction, even a "harder" and "softer" pomeron $\mathbb{P}$, by respectively "longer" (here $\sim 0.7 \mathrm{fm}$ ) and "shorter" (here $\sim 0.09 \mathrm{fm}$ ) diffraction. It even perhaps also merits a perspective of a "longer" and a "shorter", but nevertheless still universal, pomeron $\mathbb{P}$, which is in itself interesting when taken in conjunction with long-established, $s$ channel and therefore time-like, approaches to hadron-hadron diffraction.[7]

The preliminary results presented here do not address directly the degree to which the measurements by $\mathrm{H} 1$ and Zeus at HERA of the cross sections for the virtual-photoproduction of "quarkonia" are consistent with the flavour-blindness of chromodynamics. To do so requires a careful 
analysis of the magnitudes, as well as of the $W^{2}$ and $Q^{2}$-dependences, of these cross sections, work which is now underway. This work will also extend to include measurements of VM photoproduction $\left(Q^{2}=0\right)$ for all quark-flavours including $b$-quarkonia, to measurements at substantial 4-momentum transfer squared $t$, and to elastic and inelastic virtual-photon Compton scattering.

\section{Acknowledgements}

One of us (JD) wishes to record the indubitably beneficial influence of many comments in conversations with experimental and theoretical colleagues over what is now a quarter of a century concerning the meaning of the universality or otherwise of the Regge-asymptotic expansion, its relationship to QCD, and how to examine any such universality in the wealth of new measurements of diffractive interactions which have been possible in the kinematic domain involving $Q^{2}$ as well as $W^{2}$ at HERA. These comments have, consciously or unconsciously, influenced the distillation of his thoughts as many of the revealing measurements by the $\mathrm{H} 1$ and Zeus experiments have emerged and then secured the huge extension of the diffractive experimental landscape which electron-proton physics at the Fermi energy scale inevitably enables.

\section{References}

[1] H A Bethe and W Heitler, Proc. Roy. Soc. (London) 146A (1934) 83

[2] H1 Collaboration, Phys. Lett. B338 (1994) 507

Zeus Collaboration, Phys. Lett. B350 (1995) 120, Phys. Lett. B356 (1995) 601,

Z. Phys. C69 (1995) 39, Phys. Lett. B377 (1996) 259, Phys. Lett. B380 (1996) 220

H1 Collaboration, Nucl. Phys. B463 (1996) 3, Nucl. Phys. B468 (1996) 3, Nucl. Phys. B472 (1996) 3

Zeus Collaboration, Z. Phys. C73 (1996) 73, Z. Phys. C75 (1997) 215, Eur. Phys. J. C2 (1998) 247,

Phys. Lett. B437 (1998) 432, Eur. Phys. J. C6 (1999) 603

H1 Collaboration, Z. Phys. C75 (1997) 607, Eur. Phys. J. C10 (1999) 373,

Eur. Phys. J.C13 (2000) 371, , Phys. Lett. B483 (2000) 23, Phys. Lett.B483 (2000) 360

Zeus Collaboration, Eur. Phys. J. C12 (2000) 393, Eur. Phys. J. C14 (2000) 213

Phys. Lett. $B 487$ (2000) 273, Eur. Phys. J. C24 (2002) 3, 345, Eur. Phys. J. C26 (2003) 389,

Nucl. Phys. B695 (2004) 3, Nucl. Phys. B718 (2005) 3

H1 Collaboration, Phys. Lett. B541 (2002) 251, Phys. Lett. B568 (2003) 205,

Eur. Phys. J.C46 (2006) 585, Phys. Lett. B638 (2006) 422

Zeus Collaboration, PMC Physics A 1 (2007) 6, Phys. Lett. B680 (2009) 4

H1 Collaboration, JHEP 05 (2010) 032, Eur. Phys. J. C73 (2013) 2466

Zeus Collaboration, JHEP 05 (2010) 085, Phys. Lett. B708 (2012) 14-20, JHEP 02 (2013) 071,

Nucl. Phys. B909 (2016) 934

[3] T Regge, Nuov. Cim. 14 (1959) 951

G Chew, S Frautschi, S Mandelstam, Phys. Rev. 126 (1962) 1202

[4] PLUTO Collaboration, Phys. Lett. B142 (1984) 111, Phys. Lett. B142 (1984) 119

[5] H1 Collaboration, Phys. Lett. B598 (2004) 159

[6] L Lipatov, Sov. J. Nucl. Phys. 23 (1976) 338,

"Pomeron in Quantum Chromodynamics" ed. A H Mueller, World Scientific (1989)

E A Kuraev, L Lipatov, V S Fadin, Sov. Phys. JETP 44 (1976) 443, Sov. Phys. JETP 45 (1977) 199

L Tyburski, Phys. Rev. D13 (1976) 1107

H Cheng, C Y Lo, Phys. Rev. D13 (1976) 1131, Phys. Rev. D15 (1977) 2959

L L Frankfurt, V E Sherman, Sov. J. Nucl. Phys. 23 (1976) 581

Y Y Balitsky, L Lipatov, Sov. J. Nucl. Phys. 28 (1978) 822

[7] M L Good, W D Walker, Phys. Rev. 120 (1960) 1857 